\title{
Low-cost Implementation of IEC 61850 based Substation Automation System in Conventional Hard Wired 132 KV Class Power Substations
}

\author{
Pooja Rai \\ $M$ Tech (4th Sem), EEE Dept. \\ Bhilai Institute of Technology, Durg \\ poojarai1908@gmail.com
}

\author{
Dr. Anup Mishra \\ Professor \& Head of EEE Dept, Bhilai \\ Institute of Technology, Durg \\ anupmishra.bit123@gmail.com
}

\author{
Abhijeet Lal \\ Assistant Prof., EEE Dept, \\ Bhilai Institute of Technology, Durg \\ abhijeet.lal@bitdurg.ac.in
}

\begin{abstract}
The operation, control and protection of EHV substations have benefited immensely from advancements in electronics, computers, information and communications technology. The use of IEC61850 compliant digital protection relays, digital energy meters, smart monitoring devices and other smart interactive IEDs are being extensively used in all EHV substations. The integration and convergence of developments in computer, communication, electronics technologies with power system control and protection have revolutionized the way substations are now being operated. Nowadays, $400 \mathrm{KV}$ and above voltage class new EHV substations of Indian central and state utilities are invariably being designed with IEC61850 based SAS. However, new $132 \mathrm{KV}$ class substations are still constructed without Substation Automation System owing to substantial cost implication SAS. Further, for the same reason a majority of old EHV substations in Indian power utilities could not be provided with SAS so far. This paper presents a case study on low-cost implementation of IEC 61850 based substation automation in old and smaller conventional hard wired $132 \mathrm{KV}$ substations and thus converting them into smart substations. The paper begins with a brief description of a typical $132 \mathrm{KV}$ class substation, followed by an introduction to Substation Automation system and IEC 61850 based SAS. Thereafter, the paper discusses design aspects of implementation of economically optimized SAS in conventional hard-wired Substations and discusses benefits of implementation of SAS in $132 \mathrm{KV}$ class Substations. The paper concludes with a discussion of the benefits of adoption of IEC 61850 based substation automation system in conventional EHV substations.
\end{abstract}

Keywords - IED; SAS; RTU; IEC61850

\section{INTRODUCTION}

$132 \mathrm{KV}$ class EHV substations constitute around 70 to 75 $\%$ of EHV substations in an Indian state power utility. In Indian context, these are usually the interface substations between the transmission and distribution power state utilities. Many of these are radial substations and located in distant remote locations near load centres. In Chhattisgarh state, some of these substations are situated in troubled places such as Sukma, Bijapur, Dornapal, Pakhanjur etc. Access to these places may be tedious, time consuming and sometimes constrained by other factors. Deputing engineer/technical personnel to such substations for trouble shooting, repair, maintenance involves significant costs and time. As a consequence, the experts of the utilities cannot access the substations promptly from remote for analysis of any fault event by, which often results in delayed decision making and therefore delayed safe system restoration. The utilities providing electricity services would want to cut down on such expenses by bringing down the number of visits of an engineer or technician to a substation. In these substations, the control and relay panels are placed in a central control room. Control and protection devices have been copper wired to CTs, VTs, switchgear and switchyard devices. The state utilities are procuring IEC61850 compliant control and protection IEDs in new substations as it is the new established industry norm. In older substations also, utilities have taken up modernization programme and electromechanical/ older version static and microprocessor-based relays have either been replaced or in the process of replacement by new state of art digital IEDs which are IEC 61850 compliant. As mentioned earlier, in smaller 132 KV Substations, the utilities did not opt for a comprehensive Substation Automation System on account of huge cost implications. The SAS vendors usually promote their detailed, comprehensive, fully redundant SAS products, the cost of which become prohibitive for smaller substations and therefore benefits of automation system are not available to majority of these. This paper presents a case study on low-cost implementation of IEC 61850 based substation automation system (SAS) in these substations utilizing already available resources there. The cost curtailment is achieved by utilizing existing BPUs as BCUs, existing smart RTU and optimizing required communication infrastructure. In these substations, the Bay Protection Units and various interlocks are hardwired. The conventional control and relay panel setup is centralized and placed in the control room. Redundancy in IEDs and communication setup is optimized without compromising critical aspects of SAS, thereby affecting considerable cost reduction.

A typical $132 \mathrm{KV}$ class substation consists of two numbers of $40 \mathrm{MVA}, 132 / 33 \mathrm{KV}$ Power transformers, 5 to 7 numbers $132 \mathrm{KV}$ bays on one main and one transfer bus scheme and 7 to 10 Numbers of $33 \mathrm{KV}$ feeder bays on one main and one transfer bus scheme. 


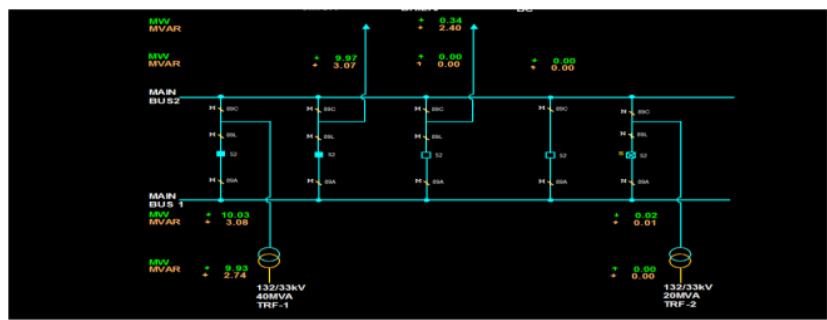

Fig. 1. 132 KV Side of SLD of a typical 132/33 KV Substation

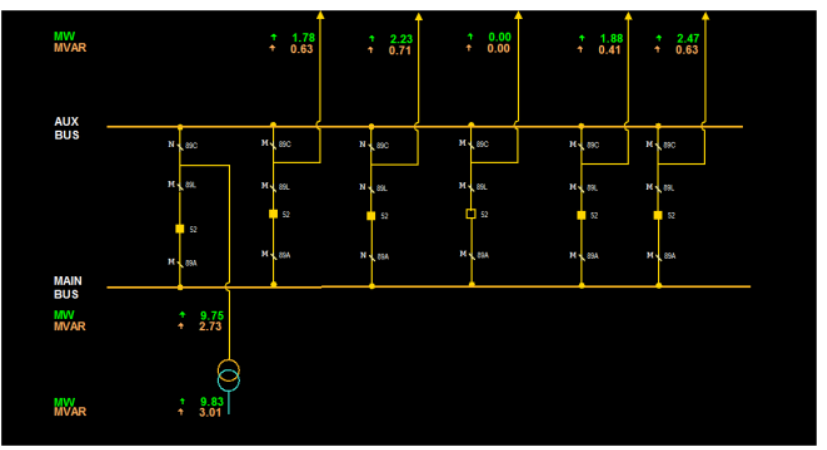

Fig. 2. $33 \mathrm{KV}$ side of SLD of a typical 132/33 KV S/s

\section{INTRODUCTION TO SUBSTATION AUTOMATION SYSTEM}

Electric power substation can be controlled and and monitored from a central work station HMI with the help of Substation Automation System. The use of Digital relays, digital energy meters, monitoring devices and other smart communicable IEDs have brought about tremendous changes in substation functioning.

This low-cost SAS contains the following main components:

(a) Bay Protection units as Bay Control Unit IEDs for protection, control and monitoring.

(b) Industrial grade, Station Human Machine Interface (HMI) Server cum Engineering Work Station

(c) Remote terminal Unit as communication Gateway to Remote Control Centre \& SLDC

(d) Ethernet Local Area Network infrastructure

(e) Peripheral equipment like printers, GPS along with Visual Display Unit etc.

SAS empowers local control Centre to monitor and operate substation elements with the help of human machine interface (HMI). This software interface contains an extensive range of supervisory control and data acquisition (SCADA) functions. The communication gateway facilitates the information flow with Remote Control Centre. The BPU (Bay protection unit) and BCU (Bay control unit) IEDs provide connection to Circuit Breakers and Disconnectors. Monitoring, control and protection functions are performed through these BCU \& BPU.

\section{BRIEF DESCRIPTION OF IEC 61850}

The contents of IEC 61850 are presented in brief hereunder;

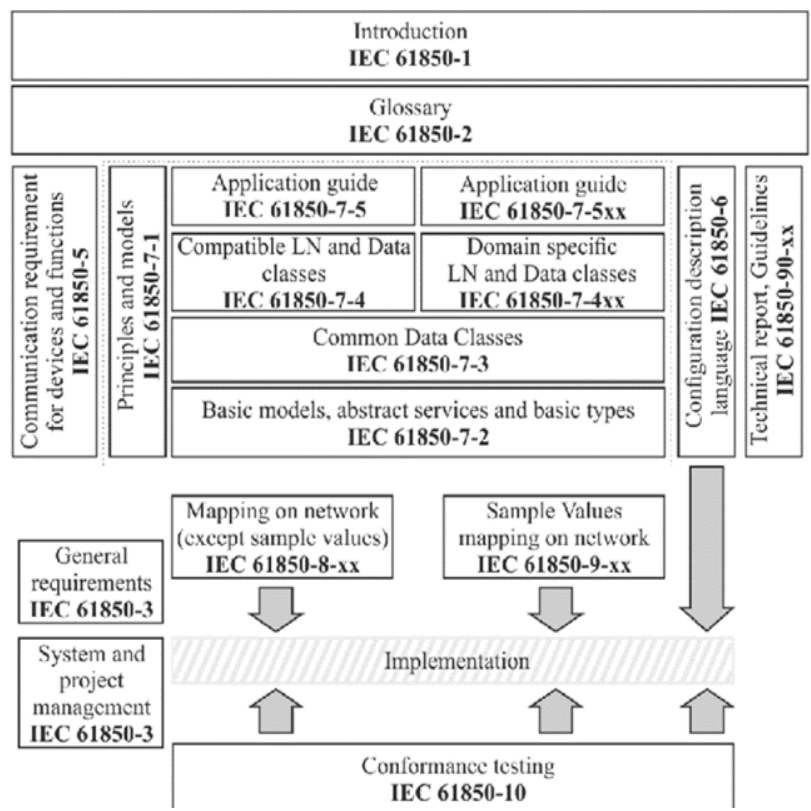

Fig. 3. IEC 61850 Contents

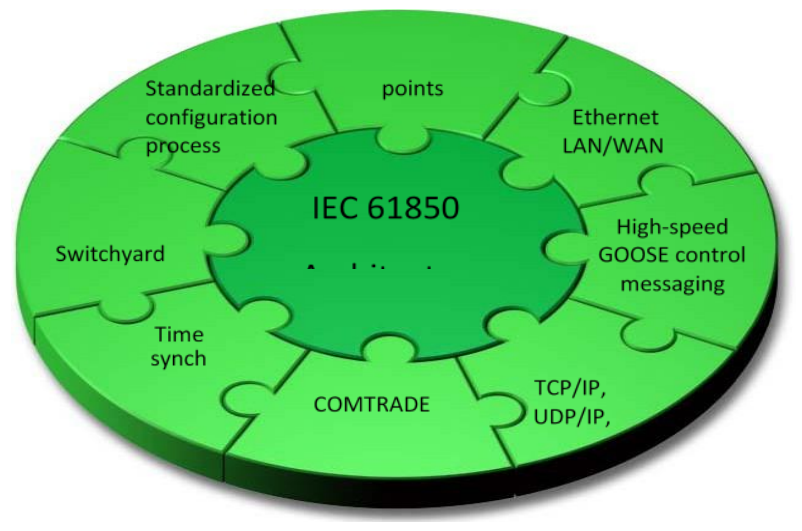

Fig. 4. Pictorial representation of multitasking achieved with IEC 61850.

IEC 61850 family of standards specify data models of a wide range of substation elements which facilitate system operators to achieve smooth communication between devices of different vendors.

In IEC 61850 standard, a physical device is virtualized into a Logical Device (LD). A logical device has many Logical Nodes (LN). The smallest part of a data sharing function is a logical node (LN). Each information is logically organized and named e.g. the LN "XCBR" represents a circuit breaker \& the LN "MMXU" represents measurement of power, voltage, current and impedance. A Logical Node comprises of many standardized Data Objects (DO). 


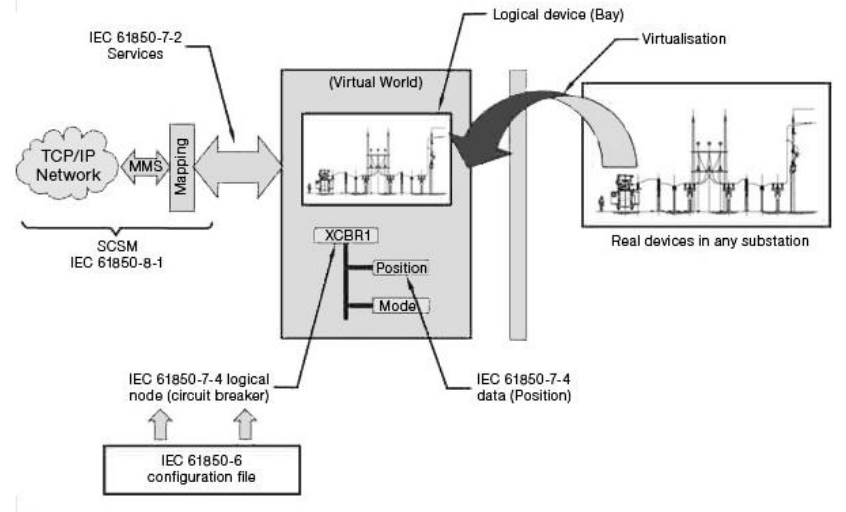

Fig. 5. The modeling concept of IEC 61850.

The IEC 61850 object model environment supports read/write functions, reporting and command execution, processing of events and many other services. Ethernet with TCP/IP is the key protocol used in IEC 61850 and the communication is carried out through a client server mode. The client initiates the connection. Many services demand prompt execution. The GOOSE (Generic Object-Oriented Substation Event) messages allow expedited delivery as these have a high priority tag. TCP/IP stack do not handle GOOSE frames. These are communicated through a publisher-subscriber mechanism in a 5 to $10 \mathrm{~ms}$ time margin. The same method is applicable for SMV (Sampled Measured Values). The IEC 61850 standard offers support beyond data models, abstract communication services and technology mappings. The environmental conditions for IEDs are specified in system aspects whereas the part 10 of IEC61850 defines testing procedures to ensure high conformance level.

In this family of standards, all information exchanged in a substation communication network are defined by Substation Configuration Language (SCL). It is a programming language based on XML-based. SCL defines a set of formats containing the device model (logical nodes, communication systems and functionalities). The SCL is used to create many files for comprehensive depiction of the IED. Each SCL file contains Header which identifies configuration and version of an SCL file. The header also defines options for the mapping of names to signals. SCL files contain Substation description, IED description, Communication system description and L Node Type definitions as well.

The substation automation utilizes following SCL files:

- IED Capability Description (ICD file) defines the capabilities of the IED and the pre- configured data model of the IED.

- Configured IED Description (CID file) describes an instantiated IED with all the relevant configuration parameters.

- Substation Specification Description (SSD) file contains the single line diagram of the substation and its LNs and describes the power system functions of SAS.
- Substation Configuration Description (SCD) file specifies the configuration of a substation in detail. The SCD file comprises of several ICD files and one SSD file.

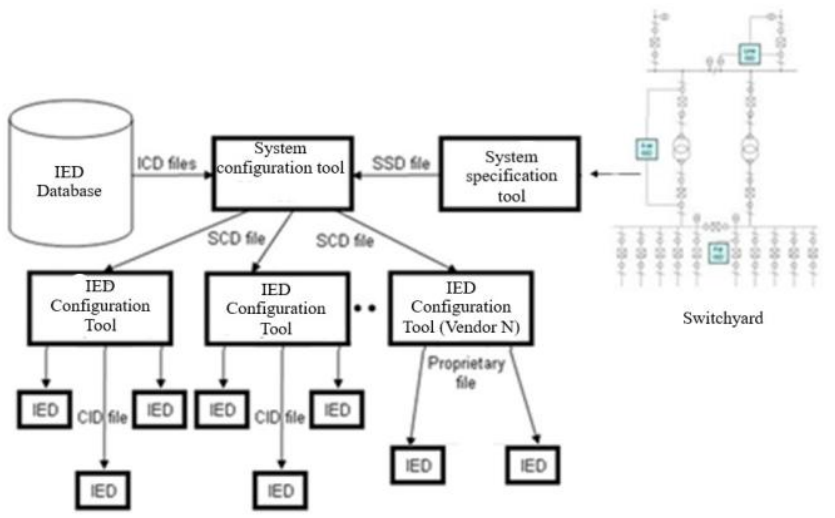

Fig. 6. SCL files of IEC 61850

\section{IEC 61850 BASED SUBSTATION AUTOMATION ARCHITECTURE}

Typical schematic of an IEC 61850 based substation automation system is presented below.

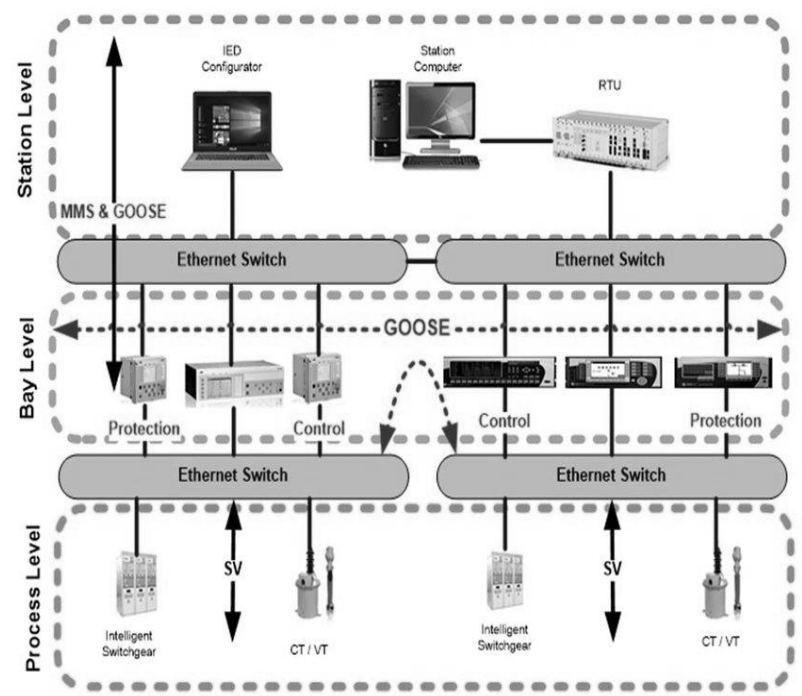

Fig. 7. IEC 61850 Substation automation system schematic

The following figure shows the process and station buses in relation to management of switchyard and entire substation activities; SAS comprises of three hierarchical levels, viz. Bay Level, Process level and Station level. The station level consists of IEDs at the station, Remote Control Center or SCADA at Load Dispatch Centre. The bay level comprises of main IEDs, viz. bay control units (BCUs), bay protection units (BPUs), phasor measurement units (PMUs), Measuring Centers (MCs) etc. The process level connects the power system element with SAS. The Station Level functions refer to the substation as a whole. Bay level functions act on the primary equipment of that bay. The Process level functions interface to the process, i.e. basically binary and analog $\mathrm{I} / \mathrm{O}$ 
functions like data acquisition and issuing commands. IEC 61850 standards envisage two buses viz. the station bus and the process bus on the Ethernet, TCP/IP technology. Station bus and Process bus share ethernet switches in the same communication network. The IEC 61850 Station Bus links all bays with the station supervisory level and carries control in a particular order such as measurement, interlocking and operations. Use of GOOSE (Generic Object- Oriented Substation Events) messages for fast transfer of critical protection information are implemented by the station bus. Substantial reduction is achieved in Copper wiring which has been traditionally used for conveying binary information between protection IEDs/relays. Process Bus provides digital interconnection to primary substation equipment viz. switchgear and instrument transformer. Application of process bus substantially reduces conventional copper wiring inside the substation switchyard. It also eliminates the high energy signals. The station bus connects the protection, control and monitoring IEDs (i.e. bay units) with the station computer having HMI (i.e. station level devices) and the gateway to the communication center. The process bus interconnects the bay units with the switchyard devices.

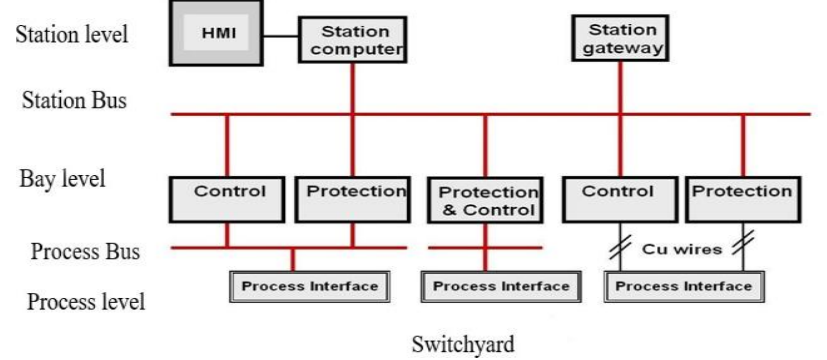

Fig. 8. IEC 61850 based Substation Automation System Process \& Station buses

\section{CONTROL AND PROTECTION FACILITIES AT A TYPICAL 132/33 KV S/S}

These are conventional copper wired substation and all the control and relay panels are placed in a control room. New substations are provided with IEC61850 compliant digital protection units and in older substations, most of the utilities have replaced the older generation static/ microprocessor-based relays by IEC 61850 compliant digital protection relays (IEDs) in past few years. The analog inputs from CT/VTs are copper wired to IEDs in their respective Control and Relay panels. The circuit breaker status signals are copper wired to protection relays. The disconnectors are generally not motorized. The status signals of switchgear are brought to control panels for conventional semaphore and LED indications. Smart digital sensors are employed in Power transformers for monitoring of oil and other parameters. Presently, in absence of automation, the protection relay indications are recorded manually in these substations. Various periodical instrumentation and metering data are also recorded and processed manually in log sheets for further analysis. Manual recording and processing of data is very tedious, time consuming and error prone and many a times it results in delayed response to system fault occurrences. Some of the substation auxiliaries are equipped with smart sensors. The details of IEC61850 compliant protection IEDs and other control/protection facilities available in the C\&R panels of the said substation is presented in tabular form;

\section{TABLE I. THE DETAILS OF IEC61850 COMPLIANT PROTECTION IEDS}

\begin{tabular}{|c|c|c|c|c|c|c|c|c|}
\hline \multirow{2}{*}{$\begin{array}{l}\text { S } \\
\text { No }\end{array}$} & \multirow{2}{*}{$\begin{array}{c}\text { Protection } \\
\text { Schemes }\end{array}$} & \multicolumn{2}{|c|}{$\begin{array}{c}40 \text { MVA } \\
\text { Transformer }\end{array}$} & \multirow{2}{*}{$\begin{array}{c}\text { 132KV } \\
\text { Feeder } \\
\text { Bay }\end{array}$} & \multirow{2}{*}{$\begin{array}{c}132 \mathrm{KV} \\
\text { Transfer } \\
\text { Bay }\end{array}$} & \multirow{2}{*}{$\begin{array}{c}33 \mathrm{KV} \\
\text { feeder } \\
\text { bay }\end{array}$} & \multirow{2}{*}{\begin{tabular}{|c|}
$33 \mathrm{KV}$ \\
Bus \\
Transfer \\
Bay
\end{tabular}} & \multirow{2}{*}{$\begin{array}{c}33 \mathrm{KV} \\
\text { Capacitor } \\
\text { Bank bay }\end{array}$} \\
\hline & & $\begin{array}{c}132 \mathrm{KV} \\
\text { Side }\end{array}$ & $\begin{array}{c}33 \mathrm{KV} \\
\text { Side }\end{array}$ & & & & & \\
\hline 1 & Differential relay & 2 & Nil & Nil & Nil & Nil & Nil & Nil \\
\hline 2 & Distance Relay & Nil & Nil & 1 & Nil & 1 & Nil & Nil \\
\hline 3 & Over fluxing relay & 1 & Nil & Nil & Nil & Nil & Nil & Nil \\
\hline 4 & LBB relay & 1 & 1 & 1 & 1 & 1 & 1 & 1 \\
\hline 5 & $\begin{array}{l}\text { Trip circuit } \\
\text { supervision relay }\end{array}$ & 2 Nos & 2 Nos & 2 Nos & 2 Nos & 2 Nos. & 2 Nos. & 2 Nos. \\
\hline 6 & $\begin{array}{l}110 \mathrm{~V} \mathrm{DC} \\
\text { control voltage } \\
\text { monitoring relay }\end{array}$ & 2 Sets & 2 Sets & 2 Sets & 2 Sets & 2 Sets & 2 Sets & 2 Sets \\
\hline 7 & $\begin{array}{l}\text { Neutral } \\
\text { displacement relay }\end{array}$ & Nil & Nil & Nil & Nil & Nil & Nil & 1 \\
\hline 8 & $\mathrm{O} / \mathrm{C}$ and $\mathrm{E} / \mathrm{F}$ relay & 1 & 1 & 1 & 1 & 1 & 1 & 1 \\
\hline 9 & $\begin{array}{l}\text { Over load } \\
\text { Protection } \\
\end{array}$ & 1 & Nil & Nil & Nil & Nil & Nil & Nil \\
\hline 10 & $\begin{array}{l}\text { Lock out trip relay } \\
\text { Electrically reset } \\
\text { Type }\end{array}$ & 2 Sets. & 2 Sets & 2 Sets & Nil & 2 Sets & 2 Sets & 2 Sets \\
\hline 11 & $\begin{array}{l}\text { Transformer body } \\
\text { protection relays. }\end{array}$ & 1 Set & Nil & Nil & Nil & Nil & Nil & $1 \mathrm{set}$ \\
\hline 12 & REF Relay & 1 & Nil & Nil & Nil & Nil & Nil & Nil \\
\hline 13 & $\begin{array}{l}\text { Trip transfer } \\
\text { switch }\end{array}$ & 1 & 1 & 1 & Nil & 1 & Nil & Nil \\
\hline 14 & TNC switch & 1 No. & 1 No. & $1 \mathrm{No}$ & $1 \mathrm{No}$ & 1 No. & 1 No. & 1 No. \\
\hline 15 & $\begin{array}{l}\text { Tri-vector Energy } \\
\text { Meter }\end{array}$ & 1 & 1 & 1 & Nil & 1 & 1 & 1 \\
\hline
\end{tabular}

\section{IEC 61850 INFRASTRUCTURE FOR IMPLEMENTATION OF LOW-COST SAS AT SMALLER $132 \mathrm{KV} \mathrm{S} / \mathrm{S}$}

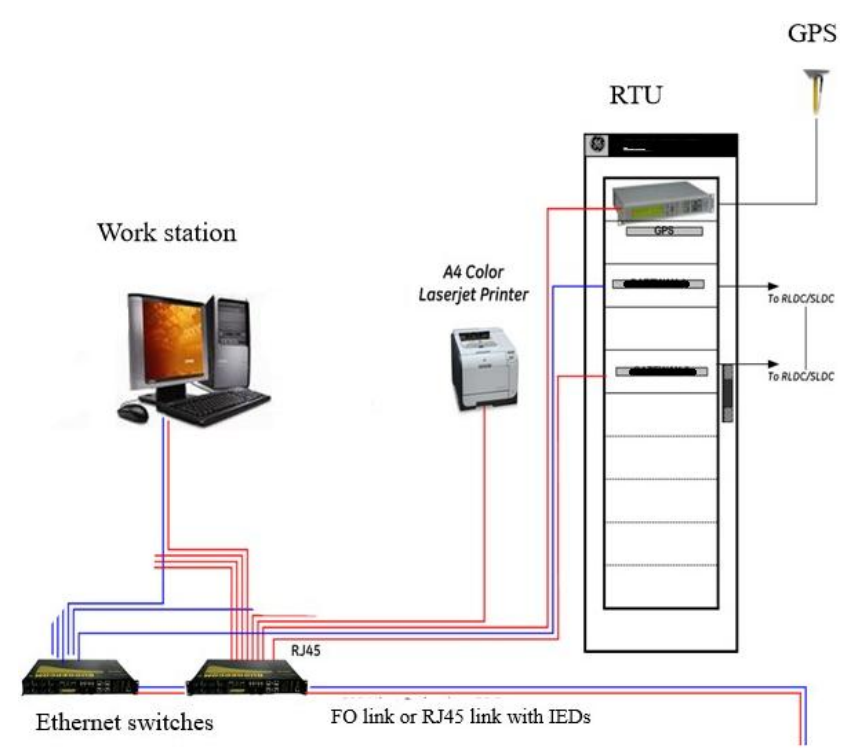

Fig. 9. Optimized SAS station HMI Equipment at Control Room.

SAS architecture of Figure 9 comprises of minimum quantity of equipment as below;

- $\quad$ Station HMI and server Station HMI cum engineering and DR Work Station: 1 No

- Any IEC61850 compliant Numerical IED as Bay control Unit for each substation Bay 
- Ethernet switches as required

- $\quad$ Existing IEC 61850 compliant RTU

- Communication infrastructure

- Colour Laser Printer (For Reports \& Disturbance records)

In this low-cost SAS scheme, Control and protection features are combined in the same IED. Further, redundancy of $\mathrm{HMI}$ at station level is reduced. This arrangement is depicted in Fig 11.

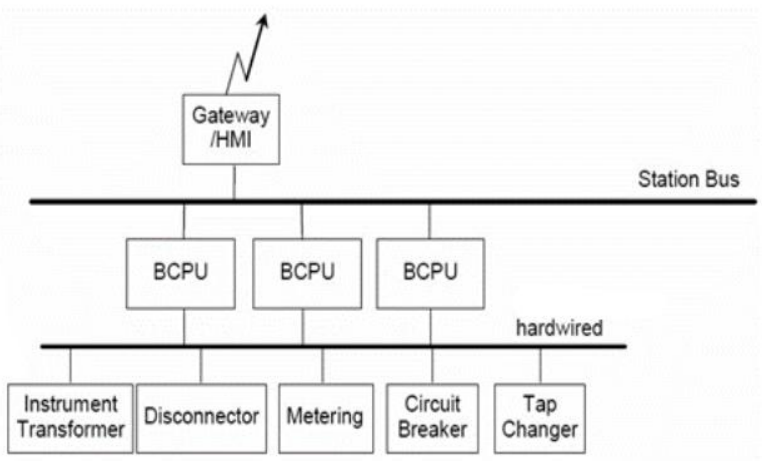

Fig. 10. Optimized SAS station HMI Equipment at Control Room

General schematic of SAS architecture is presented below showing connection of IEC 61850 based IEDs with ethernet switches, ring connection of ethernet switches, separate ring connection of energy meters which are on Modbus, protocol converter Modbus to IEC61850, station HMI with accessories, gateway to Remote Control Centre, GPS clock etc.

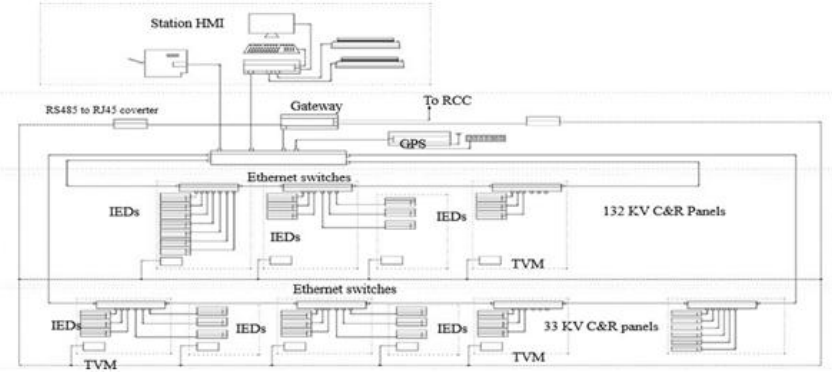

Fig. 11. Cost optimized SAS architecture

\section{SYSTEM DESIGN SPECIFICATIONS:}

Existing control and relay panels are placed in the centralized control room. As a cost saving measure, one of the bay protection units (BPU) is utilized as BCU (Bay control unit) for each bay. The Circuit Breaker closing command is wired to the BPU for control. Also, the status signals of switchgear are connected to BPU as digital inputs. SAS can support control and monitoring from Remote Control Centre via RTU. In this low-cost arrangement, SAS architecture is structured in a station and a bay level. At bay level, the available Bay Protection Unit IEDs take care of all bay level monitoring, control and protection functions including analogue measuring inputs, $\mathrm{CB} /$ Disconnector status indication and outputs for commands. The data exchanges between bay and station level IEDs take place through communication infrastructure viz. ethernet switches, LIUs, shielded copper cable \& fibre-optic cables etc. The fibre optic cables may be run in HDPE conduit pipes or GI pipes to protect these from rodents. IEC 61850 protocol is utilized for data exchange between various SAS components. Tri-vector energy meters may not be IEC61850 compliant and in such case suitable protocol converters are used to integrate these in SAS. Adequate numbers of ethernet switches with sufficient numbers of spare ports are used. The network redundancy is ensured with ring communication infrastructure.

SAS software are designed to enable operator, having little or no knowledge in computer hardware and software programming, to operate the system. With some preliminary training, operating staff can operate the system easily. The substation operating personnel can issue commands to the switchgear via mouse clicks or keyboard commands on single line diagram screen display. The scope of SAS includes monitoring, control and some specified protection functions in the instant case as the BPUs are already hardwired to switchgear and CT/PT. SAS also takes care of self-monitoring, signaling measuring as well as memory functions, event recording and disturbance recording. The work station HMI of SAS provides following standard screens;

- Single-line diagram showing the $\mathrm{CB}$ and Disconnector status and $\mathrm{P}, \mathrm{Q}, \mathrm{V}, \mathrm{I}, \mathrm{Hz}$ values (separate screens for measurements from BPUs and energy meters).

- Interlocking and blocking control dialogues details. The control dialogues indicate the entire interlock sequence of a particular switchgear operation.

- Measurement dialogues

- Alarm list

- $\quad$ Event list,

- System status

SAS provides for self-monitoring of components, modules and communication. This improves availability and the reliability of the equipment and significantly minimizes maintenance activities which can be restricted to need based maintenance.

The station HMI performs functions of control and supervision of the substation. The operator station HMI can be a redundant in hot standby mode, however single station HMI is adequate and has been so considered in this case. Alternatively, in the event of failure of SAS, the switchgear can be operated through manual switches provided in the control panels, since all the control and relay panels are placed at a central location i.e. Control room. Operation of a single switch from more than one of the many control levels, i.e. Remote Control Centre, station HMI, control panels, at the same time is prevented with the help of clear control priorities. The priority can be predefined. The GPS time synchronizing signal for the synchronization of the entire system is generally provided along with GPS at local control Centre along with Visual clock Display Unit. In the absence of high bandwidth communication link, the Remote-Control Centre can operate 
and monitor through PLCC (Power Line Carrier Communication Link) on IEC60870-101 protocol.

\section{DISCUSSION: BENEFITS OF SUBSTATION AUTOMATION SYSTEM}

Following parameters can be monitored, recorded, processed and analyzed with SAS:

- Circuit Breaker and Isolator status indication

- Voltage, current, MW, MVAR, frequency Measurement of all bays viz. feeder/ transformer bays, etc.

- Fault events (including fault current, fault time stamping and other fault data)

- $\quad$ Time stamped Disturbance records and alarms data

- Tap position of Transformer and oil parameters with smart sensors

- Power transformer's winding/oil temperature \& ambient temperature

- $415 \mathrm{~V}$ LT system and substation battery system monitoring

- Monitoring of substation auxiliaries such as fire protection system etc.

- Acquisition of all monitoring data of substation

- Acquisition of events, alarms and disturbance records from protection IEDs.

\section{A. Automatic disturbance file transfer}

Protection IEDs' event/alarm/disturbance data can be uploaded automatically (triggered on event or periodical e.g. once per day) to a station HMI/work station and be stored on the hard disc. These files can either be accessed from remote if high bandwidth communication link is available at the substation. Alternatively, these can be shared by operators with the technical experts of the utilities either on email or over other wireless platforms. This helps reduce restoration time post fault occurrence. Further, informed and timely participation of experts, even from distant locations, ensures safety and security of equipment and personnel.

\section{B. Disturbance analysis}

The event/fault records acquired from the relays can be evaluated with the help of software tool resident in station HMI for proper fault analysis. Alternatively, a separate PC based work station can be provided for this purpose.

\section{IED parameter setting}

All protection and control IEDs can be accessed for reading the parameters (settings) from the station HMI or from a separate Engineering work station. Change in protection IED parameters settings or the activation of parameter sets can be carried out by authorized personnel only and it is permitted after entering a password.

\section{Generation of Reports}

These reports include presentation of measured and calculated values for different time frames e.g. computation of mean and peak of measured value of daily, monthly, semiannually and yearly basis. Historical trend reports of selected analogue values on daily (at 15 minutes interval), Weekly, Monthly and Yearly are also available with SAS. Data of specific time period can be selected for further processing. There have been enormous developments with regard to capability of database and the memory size. Following results can be processed on demand:

- Daily MW, MVAR, Voltage and frequency curves (with respect to time, time duration $24 \mathrm{Hrs}$ ).

- Weekly/monthly real and derived analogue measurements trends.

- Processing of occurrence and duration of maximum and minimum values for $\mathrm{P}, \mathrm{Q}, \mathrm{V}, \mathrm{I}$ for each feeder/transformer on daily/weekly/ monthly basis.

- $\mathrm{CB}$ and disconnector operation data with date and time stampings.

- Shift wise and daily reports of operations and events.

- Report on selectable time frame for MW, MVAR, Current, Voltage on each feeder/transformer

- Report on selectable time for Tap Positions, winding/oil temperature, status of pumps and fans for Power transformers.

- Energy balance report of the 132 and $33 \mathrm{KV}$ buses daily and monthly basis (data from energy meters).

\section{E. Historical data trend display}

With SAS, it is possible to illustrate as trends, all types of input/output, binary and analogue process data. These graphical form or curve diagram trends can be obtained with a variety of logged value from accumulated database of SAS.

In absence of SAS, these data are logged and processed manually by operating staff of the substations. Manual execution of such work can be a tedious, time consuming work and labor intensive. Element of human error in data logging and computation cannot be ruled out. Digital recording and computing not only eliminates human drudgery but also releases precious trained manpower for maintenance and other more useful works.

\section{F. Addressing Security concerns}

Authority levels are provided for security against trespass by unauthorized user.

Each user is given rights to access specific group of functions e.g.:

- $\quad$ Access to HMI screen display only

- Open/close operation of Circuit Breaker (\& disconnector if its motorized)

- Restricted operation such as bypass of interlocks etc. 
- System administrator

Authorization levels usually provided in SAS software for engineering/maintenance purposes of the station HMI, are given below;

- $\quad$ Engineering not permitted

- Permission for Engineering/configuration

- Permission for management of entire system

The passwords assigned during the log-in routine defines the access rights of user. Only the system administrator can add/remove users and change access rights.

\section{G. Cost saving Measures}

A comprehensive Substation Automation System package for large \& critical grid EHV substations employs separate Bay control units and even redundant BCUs. SAS of these substations provides redundancy at ethernet switches, other communication infrastructure, station HMI, Gateways and other station level hardware. However, such provisions have huge cost implications and adopting comprehensive SAS package becomes cost prohibitive for smaller substations. The low-cost optimized SAS package discussed in this paper dispenses with BCUs and redundancy. Since Current transformer, Potential transformer secondaries, Circuit Breaker and switchgear interlock are hardwired to Bay Protection Units, level of redundancy in SAS can be safely optimized industrial grade hardware is utilized for SAS which not only further economizes the solution but also ensures easy procurement and maintenance. Use of all pervasive IEC 61850 compliant IEDs viz. Bay Protection Units, RTU etc. in smaller $132 \mathrm{KV}$ class is enabler for cost optimization of SAS. Further, use of capable RTU for multitasking i.e. as gateway to SLDC and critical component of SAS effects cost saving. Thus, the cost of optimized SAS discussed in this paper is expected to be less than $20 \%$ of a comprehensive SAS package.

\section{CONCLUSION}

High prohibitive cost of comprehensive SAS has prevented use of it in smaller $132 \mathrm{KV}$ substations. Design of low-cost SAS proposed in this paper will be an enabler and it will extend benefits of SAS to smaller substations. Implementation of SAS in conventional $132 \mathrm{KV}$ class substations reduce the restoration time after grid fault occurrences. The automation schemes provide an integrated approach wherein all the information is immediately available at the central location and that helps in identification, analysis and decision-making processes. Usually, these substations are located in remote places and high bandwidth data communication links are not available. However, most of these substations are covered by cell phone connectivity. Thus, the DR and event records gathered at the station HMI can be shared with the central station with the available communication links at these substations. In the event of system fault occurrences, timely guidance of the experts will not only ensure safety of equipment and personnel but also will help improve fault restoration time. Quite a few states have taken up setting up of their own independent communication network by laying OPGW on EHV transmission lines. If IEC61850 based SAS is implemented here as discussed in the paper, the high bandwidth communication OPGW link may be utilized for remote access of the IEDs/SAS of these substations. The remote engineering access and the automatic acquisition of disturbance oscillography contribute to quick analysis and decision making. Continuous monitoring of equipment permits more intelligent decisions resulting in economical maintenance. SAS can be so implemented to cover most aspects of substation activities including monitoring of low voltage substation supply, battery and battery chargers, firefighting apparatus also in addition to employing condition monitoring smart sensors of power apparatus like Power Transformers. In case of availability of high bandwidth communication link, SAS permits demand side management from State Load Dispatch Centres or any other Remote Control Centre.

\section{ACKNOWLEDGMENT}

I gratefully acknowledge the valuable guidance of Professors of Bhilai Institute of Technology, Durg (C.G.), India and support of CSPTCL officials.

\section{REFERENCES}

[1] IEC 61850: Communication Networks and Systems in Substations,

[2] PAC World Magazine,

[3] CSPTCL tenders (TR 11/22. TR15/23 et al) for construction of EHV S/s.

[4] Technical Journals at http://www.selinc.com

[5] Tetsuji Maeda, Global Product Management ABB Substation Automation Systems Baden (4th International Conference 'Power System Protection and Automation’ 21-22 November, 2007, New Delhi, India)

[6] R Suresh / V Selvaraj / R Angelson, Power Transmission \& Distribution Energy Automation, Siemens Gurgaon - Implementation of Distributed Protection \& Substation Automation System IEC 61850 Systems and Their Components

[7] Dr. Alexander Apostolov Los Angeles, Omicron -- IEC 61850 Systems and Their Components (4th International Conference 'Power System Protection and Automation’ 21-22 November, 2007, New Delhi, India)

[8] Moreno N, Flores M, Torres L, Juárez J, González D (2010) Case study: IEC 61850 as automation standard for new substations at CFE, practical experiences. SEL

[9] R. E. Mackiewicz, Member, IEEE - Overview of IEC 61850 and Benefits

[10] Sidhu TS, Yin Y (2007) Modelling and simulation for performance evaluation of IEC61850-based substation communication systems. IEEE Trans Power Delivery.

[11] Automating Electric Substations Using IEC 61850 November 2013

[12] B. Falahati, Z. Darabi, Y. Fu, and M. Vakilian, "Quantitative Modeling and Analysis of Substation Automation Systems," proceedings of the 2012 IEEE PES Transmission and Distribution Conference and Exposition (T\&D), Orlando, FL, May 2012.

[13] C. M. De Dominicis, P. Ferrari, A. Flammini, S. Rinaldi, and

[14] M. Quarantelli, "Integration of Existing IEC 61850-Based SAS Within New High- Availability Architectures," proceedings of the 2010 IEEE International Workshop on Applied Measurements for Power Systems (AMPS)

[15] M. P. Pozzuoli, "Ethernet in Substation Automation Applications Issues and Requirements," July 2007. Available: http://www.energycentral.com/.

[16] J. L. Blackburn and T. J. Domin, Protective Relaying: Principles and Applications, Fourth Edition, CRC Press, Boca Raton, FL, 2014. 